Revue de droit comparé du travail et de la sécurité sociale

3 | 2021

Droits du travail et systèmes nationaux de protection sociale au prisme de la crise sanitaire. Adaptations ou changements profonds?

\title{
Le régime juridique de l'emploi des artistes et comédiens
}

\section{Zina Yacoub}

\section{OpenEdition \\ Journals}

Édition électronique

URL : https://journals.openedition.org/rdctss/2210

DOI : $10.4000 /$ rdctss. 2210

ISSN : 2262-9815

Éditeur

Centre de droit comparé du travail et de la sécurité sociale

Édition imprimée

Date de publication : 1 novembre 2021

Pagination : 178-181

ISSN : 2117-4350

Référence électronique

Zina Yacoub, "Le régime juridique de l'emploi des artistes et comédiens », Revue de droit comparé du travail et de la sécurité sociale [En ligne], 3 | 2021, mis en ligne le 01 novembre 2021, consulté le 16 novembre 2022. URL : http://journals.openedition.org/rdctss/2210 ; DOI : https://doi.org/10.4000/ rdctss. 2210

\section{(c)}

Creative Commons - Attribution - Pas d'Utilisation Commerciale - Pas de Modification 4.0 International - CC BY-NC-ND 4.0

https://creativecommons.org/licenses/by-nc-nd/4.0/ 


\section{ZINA YACOUB}

UNIVERSITÉ DE BÉJAIA

\section{LE RÉGIME JURIDIQUE DE L'EMPLOI DES ARTISTES ET COMÉDIENS \\ DES ARTISTESET COMEDIENS}

La particularité du travail artistique invite à s'interroger sur la nécessité d'un statut juridique propre aux artistes et assimilés. L'artiste, du point de vue du droit, vacille entre deux statuts : celui du travailleur indépendant lorsqu'il travaille pour son propre compte, et celui de salarié lorsqu'il exerce pour le compte et sous les directives d'autrui. Les deux statuts peuvent aussi se cumuler dans la mesure où l'artiste, tout en étant salarié d'une entreprise (maison d'édition, télévision, théâtre...), exerce une activité dont il est le créateur, qui lui confère des bénéfices émanant directement de la vente de son œuvre ainsi que des droits artistiques inhérents à son statut d'artiste, et non à son statut de travailleur.

En droit algérien, les artistes, toutes catégories confondues, étaient restés dans l'ombre jusqu'à la promulgation du décret exécutif n²1-204 du 20/05/2021 fixant le régime spécifique des relations de travail des artistes et des comédiens. Tout en les définissant, ce texte assimile l'artiste et le comédien au travailleur (I) et organise le régime du contrat de travail et des relations de travail (II).

\section{I - L'ARTISTE ET LE COMÉDIEN ASSIMILÉS AU TRAVAILLEUR SALARIÉ}

C'est en application de l'article 4 de la loi n90-11 de 1990 relative aux relations de travail ${ }^{1}$ que le décret $n^{\circ} 21-204$ de $2021^{2}$ a été adopté, afin d'organiser les relations de travail spécifiques aux artistes et comédiens figurant dans la nomenclature des métiers dotés de régimes particuliers selon la loi susmentionnée.

Sans intervention législative, l'artiste serait considéré comme un prestataire de service indépendant ou un salarié régi par le droit commun relatif à chacun des deux statuts, ce qui fut le cas durant les trois décennies ayant suivi la promulgation de la loi $n^{\circ} 90-11$ relative aux relations de travail. Le législateur a tenu compte de la dépendance économique de cette catégorie professionnelle pour l'intégrer dans le régime du salariat, à défaut d'une subordination réelle ou suffisamment caractérisée préconisant cependant l'adoption de dispositifs spécifiques.

1 Art. 4 de la loi nº9-11 du 21/04/1990 relative aux relations de travail, JORA n¹7 du 25/04/1990, modifiée et complétée, dispose : "Nonobstant les dispositions de la présente loi et dans le cadre de la législation en vigueur, des dispositions particulières prises par voie réglementaire préciseront, en tant que de besoin, le régime spécifique des relations de travail concernant les dirigeants d'entreprises, les personnels navigants des transports aériens et maritimes, les personnels des navires de commerce et de pêche, les travailleurs à domicile, les journalistes, les artistes et comédiens, les représentants de commerce, les athlètes d'élite et de performance et les personnels de maison $»$.

2 Décret d'application $n^{\circ} 21-204$ du 20/05/2021 fixant le régime spécifique des relations de travail concernant les artistes et les comédiens, JORA n³9 du 30/05/2021. 


\section{AlgéRIE}

Le décret n²1-204 définit l'artiste (article 3) comme étant la personne qui exerce pour son compte ou pour le compte d'autrui, en contrepartie d'une rémunération, une activité ou un métier artistique, tels que définis par le Conseil national des arts et des lettres, quels que soient sa qualité et le support.

Le comédien, quant à lui, est défini comme étant la personne dont le métier est d'interpréter un rôle sur scène ou de le présenter à l'écran, ou à travers tout enregistrement, support, ou plateformes numériques.

Ces deux définitions ne tracent pas de frontières claires entre les deux professions au regard du droit du travail si ce n'est que l'artiste en tant que comédien exerce son travail sur scène. Cette distinction ne donne lieu dans le décret à aucune règle qui serait spécifique à l'un ou à l'autre. Au contraire ils sont systématiquement assimilés (ou associés), si bien qu'ils jouissent d'un seul et même régime juridique spécifique. À l'inverse de l'auteur, du sculpteur ou encore du peintre qui travaillent généralement pour leur propre compte, l'artiste de spectacle et spécialement le comédien sont intégrés dans un service organisé, ce qui révèle un indice d'une probable subordination ou du moins peut en constituer l'alternative le cas échéant.

Il convient de s'interroger sur l'intérêt d'un régime juridique spécifique pour l'artiste, notamment lorsqu'il exerce un travail, à titre permanent ou intermittent, pour le compte d'un seul employeur. Par ailleurs, si régime spécifique il y a, en quoi est-il plus avantageux que le droit commun?

\section{II - LES RELATIONS DE TRAVAIL DE L'ARTISTE ET DU COMÉDIEN}

\section{A - LES SPÉCIFICITÉS DU CONTRAT DE TRAVAIL}

Selon l'article 5 du décret, le travail artistique est exercé par les artistes ou les comédiens en vertu d'un contrat de travail à durée indéterminée ou à durée déterminée. Cette alternative sur la durée de la relation de travail peut laisser supposer que le législateur de 2021 se situe en dehors du champ de la législation de droit commun sur les CDD en ce sens que, d'une part, le choix entre les deux types de contrat semble totalement libre et, d'autre part, que le travail artistique est en soi un motif suffisant de recours au CDD. Dans le domaine artistique, le principe ne serait donc pas celui de la conclusion de contrat à durée indéterminée contrairement au droit commun ${ }^{3}$. Le recours au CDD ou au CDI dans le travail artistique est ainsi laissé au libre choix des parties au contrat.

Une autre règle inhabituelle prévue par le décret est celle qui consiste à exiger la rédaction du contrat de travail conclu entre l'artiste ou le comédien et son employeur, qu'il soit à durée indéterminée ou à durée déterminée ${ }^{4}$, alors que le principe consacré par le droit commun du travail réside dans l'obligation d'un écrit pour les seuls CDD, le contrat étant à défaut réputé être à durée indéterminée. Comment comprendre et interpréter l'exigence d'un tel écrit pour le CDI ? Il est probable que le motif se trouve dans deux des clauses devant figurer dans le contrat écrit et qui n'ont de sens que s'il s'agit d'un CDI. Ces deux clauses portent sur la nature des travaux à réaliser à titre individuel ou collectif, ainsi que

3 Art. 12 de la loi $n^{\circ} 90-11$ relative aux relations de travail.

4 Art. 6 du décret $n^{\circ} 21-204$. 
sur les périodes de réalisation du travail artistique. Une production artistique étant toujours d'une durée déterminée, un engagement à durée indéterminée supposerait d'accepter par avance de contribuer à des productions et des programmations inconnues au moment de la conclusion du contrat de travail, ce qui pourrait contrevenir à la liberté de l'artiste dans l'exercice de son art, avec toutes les conséquences que cela pourraient entraîner sur la poursuite de la relation contractuelle. Par ailleurs, le travail artistique se caractérisant par la discontinuité, voire la saisonnalité, il s'ensuit une alternance de périodes d'activité et d'inactivité. En donnant nonobstant la possibilité de conclure un CDI, le législateur satisfait à la fois l'intérêt de l'employeur qui souhaite recourir aux mêmes travailleurs à chaque période de production ou de programmation et celui de l'artiste en lui donnant la possibilité de conclure des engagements avec d'autres institutions ou employeurs, la pluriactivité étant très recherchée dans ces professions. Entendu ainsi, l'exigence d'un écrit pour le CDI vient en appui à la protection de la liberté de l'artiste dans l'exercice de son art et dans son droit à cumuler simultanément divers engagements.

Le décret $n^{\circ} 21-204$ distingue trois catégories d'artistes et comédiens :

- Les artistes et comédiens permanents : ce sont des salariés qui exercent leurs activités en vertu de contrats de travail, pour une période non limitée, et dont les activités artistiques constituent la principale source de revenus.

- Les artistes et comédiens intermittents : ils exercent leurs activités en vertu de contrats de travail pour une période limitée et font de leurs activités artistiques leur première source de revenus.

- Les artistes et comédiens occasionnels : ce sont les personnes qui, en sus de leur activité principale, réalisent sur la base de courts contrats de travail des activités artistiques, qui ne constituent pas leur principale source de revenus.

Ces catégories opèrent donc une distinction entre les artistes selon la durée du contrat de travail et la part du salaire dans les revenus globaux de l'artiste. Cette distinction sera reprise au chapitre 4 du décret pour reconnaître un droit à un congé exceptionnel non rémunéré pour les artistes et comédiens occasionnels. Ces derniers ayant une activité professionnelle à titre principal, ou dont ils tirent leur principal revenu, peuvent ainsi en vertu de leur statut d'artiste occasionnel demander à leur employeur principal de bénéficier de ce droit, dans la limite d'une durée de 3 mois, pour effectuer les travaux artistiques, le décret créant ainsi un motif de suspension du contrat de travail à disposition de tout travailleur.

Par ailleurs, le décret énumère précisément les éléments qui doivent figurer dans le contrat de travail de l'artiste ou du comédien. Le contrat doit ainsi identifier les deux parties au contrat, préciser la nature des activités artistiques réalisées à titre individuel ou collectif, la durée du contrat et les périodes de réalisation du travail artistique, le montant du salaire, la durée de la période d'essai, la durée du préavis en cas de résiliation du contrat, ainsi que les modalités de révision du contrat ${ }^{5}$.

Toutes ces dispositions témoignent de la particularité du contrat de travail de l'artiste et du comédien et sans doute d'un souci de protection à l'origine de dispositifs relativement originaux, comme celui du droit à un congé exceptionnel non rémunéré.

$5 \quad$ Art. 7 du décret $n^{\circ} 21-204$. 


\section{ALGÉRIE}

\section{B - LES SPÉCIFICITÉS DES DROITS ET OBLIGATIONS DES ARTISTES}

Sans préjudice des droits prévus par la législation et la réglementation en vigueur, le décret prévoit pour l'artiste et le comédien un ensemble de droits qui les distinguent plus ou moins des autres travailleurs régis par le droit commun du travail.

Ainsi, il est délivré à l'artiste ou au comédien une carte d'artiste leur permettant d'accéder aux avantages que leur confère leur statut. En outre, l'artiste/comédien exerce librement son travail artistique dans le respect de la loi et dispose d'un droit de propriété littéraire et artistique sur ses œuvres, dans les conditions définies par le contrat de travail et dans le cadre de la législation et de la réglementation en vigueur. Outre sa souscription au régime de la sécurité sociale, l'artiste/comédien bénéficie du droit de souscrire un contrat d'assurance complémentaire couvrant les risques exceptionnels auxquels il peut être exposé dans l'exercice de ses activités.

II convient ici aussi de s'interroger sur cette garantie supplémentaire qui traduit la possibilité d'être face à des risques particuliers inhérents à certains arts, ceux du cirque par exemple (dislocation, rhumatismes, fractures, etc.) ou des conditions d'exercice de l'art plus précaires ou plus exposés physiquement ou mentalement comme ceux de la rue. Si l'intention du législateur est louable, il est légitime de se demander si la reconnaissance de ce droit est en soi suffisante, en d'autres termes s'il n'aurait pas été plus abouti de traduire formellement ce droit en une obligation légale pour tout employeur d'artistes?

Par ailleurs, et à l'instar du droit commun du travail, le décret garantit à l'artiste et au comédien le droit à la rémunération telle que définie dans le contrat de travail. Le décret ajoute enfin le droit à la protection contre toute forme de violence et d'agression dans l'exercice de son travail ou en raison de ses œuvres artistiques, témoignant d'une reconnaissance de la prise de risque particulière de l'artiste s'exposant à des publics qui peuvent développer des attitudes d'hostilité aux œuvres présentées ou interprétées, ce qui crée des risques de violences peu connues du droit commun du travail.

Le décret précise également les obligations qui pèsent sur l'artiste ou le comédien. Ainsi, il leur incombe de se conformer aux obligations prévues par le contrat de travail et le règlement intérieur, tout en respectant l'ordre public, les bonnes mœurs et la déontologie du travail artistique. Ils se doivent aussi d'effectuer les répétitions nécessaires à la réalisation du travail artistique, conformément aux règles de l'art, en informant préalablement l'employeur ou la partie contractante des conditions requises et des moyens matériels nécessaires à la réalisation du travail.

Par conséquent, les artistes et comédiens jouissent désormais d'un régime juridique spécifique du point de l'exercice de leur art, qui prend en compte certaines des spécificités de ces métiers. Pour autant, les artistes et les comédiens bénéficient des droits des travailleurs tels qu'énumérés aux articles 5 et 6 de la loi du 21 avril 1990. À la protection du droit commun s'ajoute dorénavant celle du régime spécial institué par le décret de 2021. 